\title{
Penile cancer: incremental insights into etiology, diagnosis, staging, and management
}

\author{
Curtis A. Pettaway $\cdot$ Simon Horenblas
}

Published online: 14 January 2009

(C) Springer-Verlag 2008

Penile cancer is a rare disease in developed countries and raises several diagnostic and therapeutic dilemmas. They range from the morbidity of treating the primary penile tumor with the potential for significant loss of "quality of life," to management of the inguinal region where literally one's life could be jeopardized based upon disease burden. For this topic of World Journal of Urology, dedicated to penile cancer, we brought together a group of authors active in the field to address contemporary issues and advances in penile cancer.

There are well known differences in the incidence of penile cancer, pointing to various etiological factors. The paper of Bleeker and coworkers explores in depth the current knowledge on the epidemiology and pathogenesis. Expanding their well known work on HPV in cervical cancer, they have used their knowledge to explore the role of HPV in penile cancer. Most important is their finding of flat penile lesions as the presumed most infectious lesion, most probably responsible for the transmission of HPV.

More insight into prognostic factors in order to tailor therapy is a continuous scientific endeavor in oncological surgery with very practical consequences. The field is moving away from clinical prognosticators to molecular ones.

C. A. Pettaway $(\bowtie)$

Department of Urology and Cancer Biology,

The University of Texas MD Anderson Cancer Center, 1515 Holcombe Blvd., Unit 1373, Houston, TX 77030, USA

e-mail: cpettawa@mdanderson.org

\section{S. Horenblas}

Department of Urology, Netherlands Cancer Institute, Antoni van Leeuwenhoek Hospital, Plesmanlaan 121, 1066 CX Amsterdam, The Netherlands

e-mail: s.horenblas@nki.nl
The paper of Muneer summarizes the literature on molecular prognostic factors. Unfortunately, so far, no clearcut prognostic factor has emerged from the numerous factors assessed to date. More work is needed in this field to better categorize patients in order to tailor therapy to risk, for the present pathological prognosticators still dominate risk assessment. In elegant studies, Cubilla has shown the various growth patterns in penile cancer such as perineurial invasion, vascular invasion, and high histological grade are the most important adverse pathological prognostic factors.

While amputation with its ensuing physical and psychological consequences had been standard therapy, penis preservation is now being used more commonly. In a paper from London, combining the experience of two large referral centers, Hegarty et al. emphasize the role of penis preserving strategies. In $\mathrm{T} 1$ and most $\mathrm{T} 2$ tumors they give examples of a variety of penis preserving modalities aimed at maximal tissue preservation while not compromising oncological safety. In addition, radiation therapy is considered a penis preserving strategy. Crook et al. present the world literature and their personal series in this treatment modality.

The presence and extend of lymph node metastasis can drastically alter one's prognosis in penile cancer. Inguinal lymphadenectomy, while providing reliable pathologic staging, is morbid and in the setting of patients without metastasis provides no benefit. It has been recognized for some time that histopathologic factors in the primary tumor can stratify one's risk for lymph node metastasis. In their article, Ficarra et al. review the current status of the ability of such factors to stratify a patient's likelihood of inguinal metastasis. In addition, they discuss the ability of newly developed nomograms to predict both nodal status and survival based upon clinical parameters. Defining both non and minimally invasive staging modalities is critical to 
accurate staging in a low morbidity setting. Hughes et al. describe current advances and the roles of MRI, PET/CT, ultrasound and needle aspiration in assessing the inguinal region. In addition, the current status and results of Dynamic Sentinel Node Biopsy are discussed. The actual staging of penile cancer utilizing the current American Joint Committee on Cancer has been recognized as problematic as it is essentially a pathologic staging system. Thus, its relevance to the clinical scenario and patient counseling as to prognosis is questionable. Leitje et al. provide clinically useful modifications to the TNM system based upon actual data outcomes from 500 patients treated at The Netherlands Cancer Institute. They provide a compelling argument for their proposed changes.

Surgery is clearly moving toward less invasive procedures where possible that follow established concepts of disease control. Sotelo et al. provide an update on the initial development and experience with laparoscopic inguinal lymph node dissection. The goal of such techniques are to optimize inguinal staging and therapy where possible while minimizing complications related to standard open procedures. While the morbidity of the latter open techniques have decreased it is still of concern especially among patients with no inguinal metastasis. Spiess et al. review contemporary literature on inguinal lymph node dissection to define changing patterns of complications and steps to minimize their occurrence. Finally, advanced penile cancer due to bulky inguinal metastases is currently incurable with either surgery or radiotherapy utilized as single agents. Pagliaro and Crook discuss the rationale and preliminary published experience in penile cancer utilizing multimodality treatment approaches. Pivotal clinical trials in patients with other squamous malignancies are also discussed. These types of trials clearly suggest future directions for clinical research in penile cancer and the need for multi-institutional collaboration to evaluate novel therapeutic strategies in advanced disease. 